\title{
Lyapunov Stability of Planar Waves to the Reaction-Diffusion Equation with a Non-Lipschitzian Reaction Term
}

\author{
Soyeun Jung ${ }^{1}$ and Eunkyung Ko $\mathbb{D}^{2}$ \\ ${ }^{1}$ Kongju National University, Gongju-si, Chungcheongnamdo, Republic of Korea \\ ${ }^{2}$ Major in Mathematics, College of Natural Science, Keimyung University, Daegu, Republic of Korea
}

Correspondence should be addressed to Eunkyung Ko; ekko@kmu.ac.kr

Received 18 July 2021; Accepted 17 September 2021; Published 15 October 2021

Academic Editor: Maria L. Gandarias

Copyright (c) 2021 Soyeun Jung and Eunkyung Ko. This is an open access article distributed under the Creative Commons Attribution License, which permits unrestricted use, distribution, and reproduction in any medium, provided the original work is properly cited.

Extending (Drábek and Takáč 2017), we investigate the Lyapunov stability of planar waves for the reaction-diffusion equation on $\mathbb{R}^{n}, n \geq 2$, with a $\alpha$-Hölder continuous $(0<\alpha<1)$, but not necessarily smooth reaction term. We first consider an initial value problem for the equation and then construct sub- and supersolutions to the problem by a subtle modification of the planar wave. Our main result states that a bounded classical solution to the problem stays near the planar wave for all time whenever an initial data is close enough to the planar wave.

\section{Introduction}

We consider a reaction-diffusion equation on $\mathbb{R}^{n}$

$$
u_{t}=\Delta_{x} u+f(u) \text {, }
$$

where $x=\left(x_{1}, x_{2}, \cdots, x_{n}\right) \in \mathbb{R}^{n}, n \geq 2, \Delta_{x}=\partial_{x_{1}}^{2}+\partial_{x_{2}}^{2}+\cdots+\partial_{x_{n}}^{2}$, $t>0, u(x, t) \in \mathbb{R}$, and $f: \mathbb{R} \longrightarrow \mathbb{R}$. In particular, we are concerned with the stability of the planar wave of the form

$$
u(x, t)=U\left(x_{1}-c t\right), U(-\infty)=0, U(+\infty)=1,
$$

which is the most widely studied type of travelling wave to (1) with a continuously differentiable reaction term $f$ (e.g., see [1-3] and the references therein). However, in the present paper, we assume the reaction term $f$ is not necessarily Lipschitz continuous, but only $\alpha$-Hölder continuous $(0<\alpha<1)$ and one-sided Lipschitz continuous (see (H3) in Section 1.2). A typical example for a non-Lipschitzian reaction term $f$ is

$$
f(u)=c_{1} u^{\alpha_{0}}(1-u)^{\alpha_{1}}\left(u-c_{2}\right), 0 \leq u \leq 1,
$$

for some constants $\alpha_{0}, \alpha_{1} \in(0,1), c_{1} \in \mathbb{R}$, and $c_{2} \in(0,1)$, which has the singular derivatives at $u=0$ and $u=1$. Equation (1) with this type of $f$ is more realistic and has been used extensively as biological models, in particular, Fisher's model for population genetics. If $\alpha_{0}=\alpha_{1}=1$ in the reaction function (3), the product $u(1-u)$ represents the classical logistic growth of the population. The assumption requiring very large birth or death rate of the population leads to the formula $u^{\alpha_{0}}$ $(1-u)^{\alpha_{1}}$ with $\alpha_{0}, \alpha_{1} \in(0,1)$ in (3), which gives the restriction on the differentiability of $f$ (see the classical work of population genetics $[4,5]$, or $[6,7]$ for the derivation of $f$ ).

The restriction on the reaction function $f$ makes us unable to linearize equation (1) about the planar wave (2) and to use the spectral analysis which is a standard method to study of stability of travelling waves. Instead, we construct sub- and supersolutions to (1) by an appropriate modification of the planar wave $U$, and then we show that both the planar wave $U$ and a unique solution to a Cauchy problem (1) with an initial data near $U$ are trapped by the sub- and supersolutions whose difference is sufficiently small. This method has also been used in [3] to prove the stability of planar waves (2) in the Allen-Cahn equation on $\mathbb{R}^{n}, n \geq 2$, with the continuously differentiable reaction term (i.e., $\alpha_{0}=\alpha_{1}=1$ 
in (3)). They studied that the planar wave is asymptotically stable under any initial perturbations that decay at space infinity or almost periodic perturbations. In their works, the continuous differentiability of the reaction term is necessary to construct sub- and supersolutions and to obtain the convergence rate by using the idea of mean curvature flow on $\mathbb{R}^{n-1}$ (see [8] for the idea of mean curvature). The main purpose in our project is to study the stability of the planar wave in $\mathbb{R}^{n}, n \geq 2$, without the differentiability of the reaction term.

Our analysis is totally motivated by the results of Drábek and Takáč [6], showing that the long-time asymptotic behavior of solutions to an initial value problem of a onedimensional reaction-diffusion equation

$$
\begin{cases}u_{t}=u_{x x}+f(u) & \text { for }(x, t) \in \mathbb{R} \times \mathbb{R}_{+} \\ u(x, 0)=u_{0}(x) & \text { for } x \in \mathbb{R}\end{cases}
$$

with a non-Lipschitzian reaction term $f$ defined by (3). By constructing sub- and supersolutions without the differentiability of $f$, they established the convergence of a solution $u(x, t)$ to a travelling wave solution $U(x-c t)$, that is,

$$
\sup _{x \in \mathbb{R}}|u(x, t)-U(x-c t+\zeta)| \longrightarrow 0 \text { as } t \longrightarrow \infty
$$

for some spatial shift $\zeta \in \mathbb{R}$, when the initial data $u_{0}$ is close enough to $U$ as $x$ goes to $\pm \infty$. However, they restricted the model to the simple case of one space variable by assuming a habitat of a population is a one-dimensional space, for example, a long thin strip along a straight shoreline. In order to make the model to be more realistic, we extend their method to a multidimensional space and prove the planar wave is stable under small initial perturbations in $L^{\infty}\left(\mathbb{R}^{n}\right)$ with $n \geq 2$.

The purpose of this introduction is to provide information of the profile of the planar wave (2) and the precise assumptions on the reaction term $f$ and to state our main result.

1.1. The Profile of Planar Waves. In order to study of stability of the planar wave (2), we first introduce the $x_{1}$-moving coordinate with speed $c$ by setting

$$
u(x, t)=v(z, t), z=\left(z_{1}, z_{2}, \cdots, z_{n}\right)=\left(x_{1}-c t, x_{2}, \cdots, x_{n}\right),
$$

so that the planar wave can be considered as a stationary solution. In the $(z, t)$ coordinates, equation (1) reads

$$
v_{t}=\Delta_{z} v+c v_{z_{1}}+f(v)
$$

and the planar wave is then a stationary solution $v(z, t)=$ $U\left(z_{1}\right)$ that satisfies the profile equation

$$
\begin{gathered}
0=U^{\prime \prime}\left(z_{1}\right)+c U^{\prime}\left(z_{1}\right)+f\left(U\left(z_{1}\right)\right), \\
\lim _{1} \longrightarrow-\infty\left(z_{1}\right)=0 \text { and } \lim _{z_{1} \longrightarrow \infty} U\left(z_{1}\right)=1 .
\end{gathered}
$$

The existence and monotonicity of such profiles have been studied in [1, DT2]. According to Section 2 of [6], the assumption $(\mathrm{H} 1)$ stated in Section 1.2 guarantees $C^{2}$ -profiles $U: \mathbb{R} \longrightarrow[0,1]$ for some speed $c=c_{*}$. If $U\left(z_{1}\right)$ satisfies (8), then its translate $U\left(z_{1}-\zeta\right)$ also satisfies (8) for any constant $\zeta \in \mathbb{R}$. Throughout this paper, we impose the condition

$$
U(0)=s_{0}
$$

for some constant $s_{0} \in(0,1)$ that appears in (H1), so that the $C^{2}$-profile $U: \mathbb{R} \longrightarrow[0,1]$ satisfying (8) is unique for some unique speed $c=c_{*}$. Moreover, under the assumption ( $\left.\mathrm{H} 1\right)$, the profile is nondecreasing on $\mathbb{R}$ and there is an open interval $(a, b) \subseteq \mathbb{R},-\infty \leq a<0<b \leq \infty$, such that $0 \leq U\left(z_{1}\right) \leq 1$ and $U^{\prime}\left(z_{1}\right)>0$ on $(a, b)$. The asymptotic behavior of the profile is determined solely by the behavior of $f(s)$ as $s$ $\longrightarrow 0^{+}$and $s \longrightarrow 1^{-}$. It is well-known that $a=-\infty$ and $b$ $=\infty$ in the classical case, i.e., $f \in C^{1}(\mathbb{R})$. However, in our case of $f$ being non-Lipschitzian at the points $s=0$ and $s=$ 1 , one has $-\infty<a<0<b<\infty$. More precisely, a nonLipschitzian $f$ satisfies that there exist positive constants $\gamma_{0}$ and $\gamma_{1}$ such that

$$
\begin{aligned}
\lim _{s \longrightarrow 0^{+}} \frac{f(s)}{s^{\alpha_{0}}} & =-\gamma_{0}<0 \text { and } \lim _{s \longrightarrow 1^{-}} \frac{f(s)}{(1-s)^{\alpha_{1}}} \\
& =\gamma_{1}>0, \alpha_{0}, \alpha_{1} \in(0,1),
\end{aligned}
$$

(see a typical example (3) of $f$ ). It is obvious that the limits of (10) are $-\infty$ and $\infty$, respectively, in the case of $f \in C^{1}(\mathbb{R})$ (see $[6,9]$ for further details).

In summary, the planar wave $v(z, t)=U\left(z_{1}\right)$ satisfies

$$
0=U^{\prime \prime}\left(z_{1}\right)+c U^{\prime}\left(z_{1}\right)+f\left(U\left(z_{1}\right)\right), U(0)=s_{0}
$$

and there is an open interval $(a, b) \subset \mathbb{R},-\infty<a<0<b<\infty$, such that

$$
\begin{cases}U\left(z_{1}\right)=0 & \text { for }-\infty<z_{1} \leq a, \\ U^{\prime}\left(z_{1}\right)>0 & \text { for } a<z_{1}<b, \\ U\left(z_{1}\right)=1 & \text { for } b \leq z_{1}<\infty .\end{cases}
$$

1.2. Hypotheses on the Reaction Term $f$. Throughout this paper, following [6], we assume the reaction term $f$ satisfies the following:

(H1). $f: \mathbb{R} \longrightarrow \mathbb{R}$ is continuous such that $f(0)=f\left(s_{0}\right)$ $=f(1)=0$ for some $0<s_{0}<1$. Moreover, $f(s)<0$ for any $s$ $\in\left(0, s_{0}\right) \cap(1, \infty), f(s)>0$ for all $s \in(-\infty, 0) \cap\left(s_{0}, 1\right)$, and for any $0<r \leq 1$,

$$
F(r):=\int_{0}^{r} f(s) d s<0
$$

(H2). $f$ is $\alpha$-Hölder continuous $(0<\alpha<1)$. 
(H3). $f$ is one-sided Lipschitz continuous, that is, there exists a positive number $L$ such that

$f\left(s^{\prime}\right)-f(s) \leq L\left(s^{\prime}-s\right) \quad$ for any $\quad s, s^{\prime} \in \mathbb{R} \quad$ with $\quad s<s^{\prime}$

(H4). There exists a positive constant

$$
\eta_{0}<\frac{1}{4} \min \left\{s_{0}, 1-s_{0}\right\}
$$

satisfying that for any $\eta \in\left(0, \eta_{0}\right]$ there are positive constants $\delta \in(0, \eta], \mu$ and $\bar{\mu}$ such that

$$
\begin{aligned}
& \inf _{0 \leq s \leq \delta}[f(s)-f(s+q)] \geq \underline{\mu q} \quad \text { for all } \quad \mathrm{q} \in\left(0, \mathrm{~s}_{0}-2 \eta\right), \quad(16) \\
& \inf _{1-\delta \leq s \leq 1}[f(s-q)-f(s)] \geq \bar{\mu} q \text { for all } \mathrm{q} \in\left(0,1-\mathrm{s}_{0}-2 \eta\right) .
\end{aligned}
$$

As mentioned in the previous subsection, the first assumption (H1) is needed to show the existence and monotonicity of the profile $U\left(z_{1}\right)$. The last assumption (H4), referred to as the secant conditions, holds trivially if $f \in C^{1}$ $(\mathbb{R})$ is a classical bistable type and $\delta>0$ is small enough. We can also assume (H4) even for a non-Lipschitzian reaction term $f$. The reader can see Figure 1 in [6] for an example satisfying $(\mathrm{H} 4)$.

1.3. Main Result. We now state our main theorem. Theorem 1 says that if the equation (7) starts with an initial data $v_{0}(z)$ near the planar wave $U\left(z_{1}\right)$ then the solution to (7) stays near it for all time.

Theorem 1 (Lyapunov stability). Let the assumptions (H1)-(H4) hold and $n \geq 2$. Suppose that a function $v_{0}(z) \in$ $L^{\infty}\left(\mathbb{R}^{n}\right)$ satisfies that for all $z \in \mathbb{R}^{n}$,

$$
0 \leq v_{0}(z) \leq 1 \text { and }\left|v_{0}(z)-U\left(z_{1}\right)\right|<E_{0}
$$

where $E_{0}>0$ is sufficiently small. Then, there is a unique bounded classical solution $v(z, t)$ to (7) with an initial data $v_{0}(z)$ such that for all $z \in \mathbb{R}^{n}$ and all time $t>0$,

$$
0 \leq v(z, t) \leq 1 \quad \text { and } \quad\left|v(z, t)-U\left(z_{1}\right)\right|<C E_{0}
$$

for some constant $C>0$.

Remark 2. Theorem 1 is the extension of the onedimensional stability result (Proposition 4.1) of [6] to a multidimensional space. Our assumption for the profile $U(0)$ $=s_{0}$ gives $\zeta=0$ in their result.

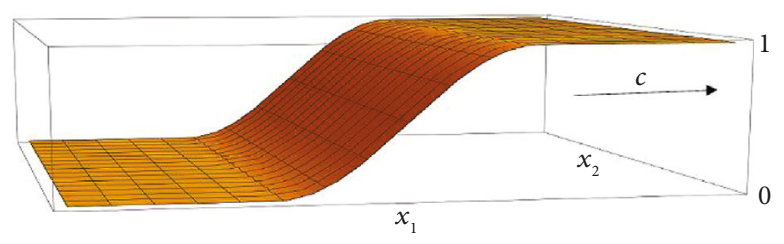

Figure 1: Planar wave $U\left(x_{1}-c t\right)$ on $\mathbb{R}^{2}$.

The paper is organized as follows. In Section 2, we first consider the Cauchy problem

$$
\begin{gathered}
v_{t}=\Delta_{z} v+c v_{z_{1}}+f(v), \\
v(z, 0)=v_{0}(z),
\end{gathered}
$$

with an initial data $v_{0}(z) \in L^{\infty}\left(\mathbb{R}^{n}\right), n \geq 2$. Under the assumption (H2), we establish regularity estimates so that a bounded mild solution to (20) is well-defined, and it becomes a bounded classical solution to (20). In Section 3, we define weak sub- and supersolutions to (20) and discuss the weak comparison principle for them under the assumption (H3). This section will also show the uniqueness of the solution to (20). Finally, in Section 4, using the assumption (H4), we modify the planar wave $U$ to construct $L^{\infty}$ suband supersolutions to (20) and prove Theorem 1 by showing both the solution to (20) and the planar wave $U$ stay between sub- and supersolutions when the initial data $v_{0}(z)$ is close enough to $U$.

1.4. Discussion and Open Problems. Theorem 1 does work if $E_{0}$ is sufficiently small that $0<E_{0}<\eta_{0}<1 / 4 \min \left\{s_{0}, 1-s_{0}\right\}$. We use the assumption (H4) to prove suitable modifications of the planar wave $U\left(z_{1}-\xi(t)\right)-q(t)$ and $U\left(z_{1}+\xi(t)\right)+q$ $(t)$ are sub- and supersolutions of (20) for some functions $\xi(t)$ and $q(t)$. By setting $q(0)=2 E_{0}$ and $\eta=E_{0}$ in (H4), we can apply the inequalities (16) and (17) to the proof of Theorem 1.

The main idea of our work follows [6], but in the present paper, we do not impose one of their assumptions, saying (H5). The assumption (H5) in [6] means that the initial perturbation is small enough only at space infinity, so the assumption (18) for an initial data implies their assumption (H5). Indeed, they proved the travelling wave is asymptotically stable by showing the solution of (20) converges to the travelling wave as $t$ goes to infinity when the initial data satisfies (H5) (see (5)). So the initial perturbation does not need to be small in their work. However, unfortunately, the assumption (H5) is not enough to prove the convergence, even the stability, for our multidimensional case. It seems that we need more assumptions on the initial perturbation or the reaction term $f$ to prove the asymptotic stability of the planar wave on $\mathbb{R}^{n}$ with $n \geq 2$, which is a interesting open problem.

Moreover, even in $\mathbb{R}$, the convergence rate for a nonLipschitzian reaction term $f$ has not been proven yet. The asymptotic stability with the convergence rate of the planar wave (2) on $\mathbb{R}^{n}, n \geq 2$, for a continuously differentiable $f$ has been studied in [3]. They also modified the planar wave 
to construct sup- and supersolutions by using more delicate phase functions thanks to the differentiability of $f$. The study of convergence rate on $\mathbb{R}$ for a non-Lipschitzian $f$ would be another very interesting direction to carry out.

\section{A Bounded Classical Solution}

In this section, we consider the Cauchy problem on $\mathbb{R}^{n}$,

$$
\begin{aligned}
v_{t} & =\Delta_{z} v+c v_{z_{1}}+f(v), \\
v(z, 0) & =v_{0}(z),
\end{aligned}
$$

where $z=\left(z_{1}, z_{2}, \cdots, z_{n}\right), n \geq 2$, an initial data $v_{0}: \mathbb{R}^{n} \longrightarrow \mathbb{R}$ is Lebesgue-measurable, and $0 \leq v_{0}(z) \leq 1$ for all $z \in \mathbb{R}^{n}$. Especially, under the assumption $(\mathrm{H} 2)$, we prove the existence of a bounded classical solution to (21) in the sense that a mild solution $v$, defined in (25), of (21) satisfies

$$
v_{t}, v_{z_{1}}, \Delta_{z} v \in C^{\alpha, \alpha / 2}\left(\mathbb{R}^{n} \times[\tau, T]\right),
$$

for any given $0<\tau<T<\infty$ and the weak star limit $v(z, t)$ * $v_{0}(z)$ in $L^{\infty}\left(\mathbb{R}^{n}\right)$ as $t \longrightarrow 0^{+}$. Here, for any $\alpha \in(0,1)$ and $T$ $>0$, the norm of Hölder space $C^{\alpha, \alpha / 2}\left(\mathbb{R}^{n} \times[0, T]\right)$ is given by

$$
\|g\|_{C^{\alpha, \alpha / 2}\left(\mathbb{R}^{n} \times[0, T]\right)}:=\|g\|_{L^{\infty}\left(\mathbb{R}^{n} \times[0, T]\right)}+[g]^{(\alpha, \alpha / 2)},
$$

where

$$
\begin{aligned}
{[g]^{(\alpha, \alpha / 2)}:=} & \sup _{z, z^{\prime \in \mathbb{R}^{n}, 0<\left|z-z^{\prime}\right| \leq 1, t \in[0, T]}} \frac{\left|g(z, t)-g\left(z^{\prime}, t\right)\right|}{\left|z-z^{\prime}\right|^{\alpha}} \\
& +\sup _{z \in \mathbb{R}^{n}, 0 \leq t<t^{\prime} \leq T} \frac{\left|g(z, t)-g\left(z, t^{\prime}\right)\right|}{\left|t-t^{\prime}\right|^{\alpha / 2}} .
\end{aligned}
$$

Applying Duhamel's principle to (21) yields an integral equation of $v$ :

$$
\begin{aligned}
v(z, t)= & \int_{\mathbb{R}^{n}} G(z-y, t) v_{0}(y) d y \\
& +\int_{0}^{t} \int_{\mathbb{R}^{n}} G(z-y, t-s) f(v(y, s)) d y d s \\
= & {\left[\mathscr{G}_{1}(t) v_{0}\right](z)+\left[\mathscr{G}_{2}(f \circ v)\right](z, t), }
\end{aligned}
$$

for any $(z, t) \in \mathbb{R}^{n} \times[0, \infty)$. Here, for any $y=\left(y_{1}, y_{2}, \cdots, y_{n}\right)$, the heat kernel $G$ is defined by

$$
G(z-y, t):=\frac{1}{(4 \pi t)^{n / 2}} e^{-\left|z_{1}-y_{1}+c t\right|^{2}+\left|z_{2}-y_{2}\right|^{2}+\cdots+\left|z_{n}-y_{n}\right|^{2} / 4 t}
$$

It is well known that if the initial value problem (21) has a solution, this solution is given by (25), referred to as the mild solution of (21). However, it is not trivial that every mild solution is a classical solution. So the first step is to establish the regularity estimates (22) for any bounded mild solution and then we will consider well-posedness of a bounded mild solu- tion (25). The estimates of the heat potentials in Hölder norm have been established in Section 4 of [10], which lead to the following lemma.

Lemma 3 (Regularity estimates). Assume that $f: \mathbb{R} \longrightarrow \mathbb{R}$ satisfies the condition (H2). If $v(z, t)$ is a bounded mild solution of (21), then for any given $0<\tau<T<\infty$

$$
v_{t}, v_{z_{1}}, \Delta_{z} v \in C^{\alpha, \alpha / 2}\left(\mathbb{R}^{n} \times[\tau, T]\right)
$$

and we have the estimates

$$
\begin{aligned}
& \left\|\left(v_{t}, v_{z_{1}}, \Delta_{z} v\right)(z, t)\right\|_{C^{\alpha, \alpha / 2}\left(\mathbb{R}^{n} \times[\tau, T]\right)} \\
& \quad \leq M_{\tau, T}\left(\|v\|_{L^{\infty}\left(\mathbb{R}^{n} \times(0, T)\right)}+\|f \circ v\|_{L^{\infty}\left(\mathbb{R}^{n} \times(0, T)\right)}\right),
\end{aligned}
$$

for some constant $M_{\tau, T}>0$, dependent upon $\tau$ and $T$.

Proof. We first fix any $\tau$ and $T$ with $0<\tau<T<\infty$. A direct calculation gives that for all $(z, t) \in \mathbb{R}^{n} \times(0, T)$,

$$
\begin{aligned}
& \left|\frac{\partial^{k+m_{1}+m_{2}+\cdots+m_{n}}}{\partial t^{k} \partial z_{1}^{m_{1}} \partial z_{2}^{m_{2}} \cdots \partial z_{n}^{m_{n}}}\left[\mathscr{G}_{1}(t) v_{0}\right](z)\right| \\
& \quad \leq M_{k, m_{1}, \cdots, m_{n}} t^{-k-m_{1}+m_{2}+\cdots+m_{n} / 2}\left\|v_{0}\right\|_{L^{\infty}\left(\mathbb{R}^{n}\right)},
\end{aligned}
$$

and for all $(z, t),\left(z, t^{\prime}\right) \in \mathbb{R}^{n} \times[0, T]$ with $t \neq t^{\prime}$ and all $i=1$ $, 2, \cdots, n$,

$$
\begin{aligned}
& \left|\frac{\partial}{\partial z_{i}} \mathscr{G}_{2}(f \circ v)(z, t)\right|, \frac{\left|\mathscr{G}_{2}(f \circ v)\left(z, t^{\prime}\right)-\mathscr{G}_{2}(f \circ v)(z, t)\right|}{\left|t^{\prime}-t\right|^{1 / 2}} \\
& \quad \leq M_{T}\|f \circ v\|_{L^{\infty}\left(\mathbb{R}^{n} \times(0, T)\right)} .
\end{aligned}
$$

The second inequality of (30) is proved in Chapter 4 of [10]. We now fix $t_{0}$ with $0<t_{0}<\tau$ and replace the initial time $t=0$ by $t=t_{0}$. By (29) and the Hölder estimates in [10], we have for all $i=1,2, \cdots, n$,

$$
\begin{aligned}
& \left\|\left(\left[\mathscr{G}_{1}(t) v_{0}\right]_{t},\left[\mathscr{G}_{1}(t) v_{0}\right]_{z_{1}},\left[\mathscr{G}_{1}(t) v_{0}\right]_{z_{i} z_{i}}\right)(z, t)\right\|_{C^{\alpha, \alpha / 2}\left(\mathbb{R}^{n} \times[\tau, T]\right)} \\
& \quad \leq M_{t_{0}, \tau, T}\left\|v\left(\cdot, t_{0}\right)\right\|_{L^{\infty}\left(\mathbb{R}^{n}\right)} \text { and } \\
& \left\|\left(\left[\mathscr{G}_{2}(f \circ v)\right]_{t},\left[\mathscr{G}_{2}(f \circ v)\right]_{z_{1}},\left[\mathscr{G}_{2}(f \circ v)\right]_{z_{i} z_{i}}\right)(z, t)\right\|_{C^{\alpha, \alpha / 2}\left(\mathbb{R}^{n} \times[\tau, T]\right)} \\
& \quad \leq M_{\tau, T}\|f \circ v\|_{C^{\alpha, \alpha / 2}\left(\mathbb{R}^{n} \times\left[t_{0}, T\right]\right)}
\end{aligned}
$$

Since $\left\|v\left(\cdot, t_{0}\right)\right\|_{L^{\infty}\left(\mathbb{R}^{n}\right)} \leq\|v\|_{L^{\infty}\left(\mathbb{R}^{n} \times(0, T)\right)}$, to obtain the estimate (28), it is enough to show that

$$
\|f \circ v\|_{C^{\alpha, \alpha / 2}\left(\mathbb{R}^{n} \times\left[t_{0}, T\right]\right)}\|f \circ v\|_{L^{\infty}\left(\mathbb{R}^{n} \times(0, T)\right)} .
$$


We notice that the inequalities (29) and (30) yield that for all $(z, t),\left(z, t^{\prime}\right) \in \mathbb{R}^{n} \times\left[t_{0}, T\right]$ with $t \neq t^{\prime}$,

$$
\begin{aligned}
& |\nabla v(z, t)|, \frac{\left|v\left(z, t^{\prime}\right)-v(z, t)\right|}{\left|t^{\prime}-t\right|^{1 / 2}} \\
& \quad \leq M_{t_{0}, T}\left(\left\|v\left(\cdot, t_{0}\right)\right\|_{L^{\infty}\left(\mathbb{R}^{n}\right)}+\|f \circ v\|_{L^{\infty}\left(\mathbb{R}^{n} \times(0, T)\right)}\right) .
\end{aligned}
$$

Since $f$ is $\alpha$-Hölder continuous, the estimates (32) give

$$
\begin{aligned}
& \sup _{0<\left|z-z^{\prime}\right| \leq 1, t \in\left[t_{0}, T\right]} \frac{\left|(f \circ v)(z, t)-(f \circ v)\left(z^{\prime}, t\right)\right|}{\left|z-z^{\prime}\right|^{\alpha}} \\
= & \sup _{0<\left|z-z^{\prime}\right| \leq 1, t \in\left[t_{0}, T\right]} \frac{\left|(f \circ v)(z, t)-(f \circ v)\left(z^{\prime}, t\right)\right|}{\left|v(z, t)-v\left(z^{\prime}, t\right)\right|^{\alpha}} \\
& \cdot \frac{\left|v(z, t)-v\left(z^{\prime}, t\right)\right|^{\alpha}}{\left|z-z^{\prime}\right|^{\alpha}} \\
\leq & M_{\tau, T}\left(\|v\|_{L^{\infty}\left(\mathbb{R}^{n} \times(0, T)\right)}+\|f \circ v\|_{L^{\infty}\left(\mathbb{R}^{n} \times(0, T)\right)}\right)
\end{aligned}
$$

and similarly

$$
\begin{aligned}
\sup _{z \in \mathbb{R}^{n}, t_{0} \leq t<t^{\prime} \leq T} \frac{\left|(f \circ v)(z, t)-(f \circ v)\left(z, t^{\prime}\right)\right|}{\left|t-t^{\prime}\right|^{\alpha / 2}} & \\
= & \sup _{z \in \mathbb{R}^{n}, t_{0} \leq t<t^{\prime} \leq T} \frac{\left|(f \circ v)(z, t)-(f \circ v)\left(z, t^{\prime}\right)\right|}{\left|v(z, t)-v\left(z, t^{\prime}\right)\right|^{\alpha}} \\
& \cdot \frac{\left|v(z, t)-v\left(z, t^{\prime}\right)\right|^{\alpha}}{\left|t-t^{\prime}\right|^{\alpha / 2}} \\
\leq & M_{\tau, T}\left(\|v\|_{L^{\infty}\left(\mathbb{R}^{n} \times(0, T)\right)}+\|f \circ v\|_{L^{\infty}\left(\mathbb{R}^{n} \times(0, T)\right)}\right) .
\end{aligned}
$$

Finally, we have

$$
\begin{aligned}
& \left\|\left(v_{t}, v_{z_{1}}, \Delta v\right)(z, t)\right\|_{C^{\alpha, \alpha / 2}\left(\mathbb{R}^{n} \times[\tau, T]\right)} \\
& \quad \leq M_{\tau, T}\left(\|v\|_{L^{\infty}\left(\mathbb{R}^{n} \times(0, T)\right)}+\|f \circ v\|_{L^{\infty}\left(\mathbb{R}^{n} \times(0, T)\right)}\right) .
\end{aligned}
$$

Noting that $f \circ v$ is the source term of the inhomogeneous initial value problem (21), we now apply the regularity estimates (28) and Tikhonov fixed point theorem to show the well-posedness of a bounded mild solution (25).

Lemma 4. Assume that $f: \mathbb{R} \longrightarrow \mathbb{R}$ satisfies the condition (H2). If an initial data $v_{0}(z) \in L^{\infty}\left(\mathbb{R}^{n}\right)$ satisfies $0 \leq v_{0}(z) \leq$ 1 , then the initial value problem (21) has a bounded mild solution $v(z, t) \in L^{\infty}\left(\mathbb{R}^{n} \times[0, \infty)\right)$ such that $0 \leq v(z, t) \leq 1$ for all $(z, t) \in \mathbb{R}^{n} \times[0, \infty)$.
Proof. We first consider the existence of a bounded mild solution $v(z, t)$ on $\mathbb{R}^{n} \times[0, T]$, where

$$
T:=\frac{1}{\sup _{-1 \leq r \leq 2}|f(r)|}>0 .
$$

We define the locally convex vector space

$$
\mathscr{X}:=L^{\infty}\left(\mathbb{R}^{n} \times[0, T]\right) \cap C\left(\mathbb{R}^{n} \times[0, T]\right),
$$

and the closed convex subset of $\mathscr{X}$

$$
\mathscr{C}:=\left\{w \in \mathscr{X}:|w(z, t)| \leq 1 \text { for all }(\mathrm{z}, \mathrm{t}) \in \mathbb{R}^{\mathrm{n}} \times[0, \mathrm{~T}]\right\} .
$$

By recalling a mild solution (25), let us consider an operator $\mathscr{F}: X \longrightarrow \mathscr{X}$ defined by

$$
\begin{aligned}
(\mathscr{F} w)(z, t) & :=\int_{0}^{t} \int_{\mathbb{R}^{n}} G(z-y, t-s) f(v(y, s)) d y d s, \forall(z, t) \\
& \in \mathbb{R}^{n} \times[0, T],
\end{aligned}
$$

where

$$
v(z, t)=\int_{\mathbb{R}^{n}} G(z-y, t) v_{0}(y) d y+w(z, t) .
$$

We first prove $\mathscr{F}(\mathscr{C}) \subset C$. For any given $w \in \mathscr{C}$, since $\int_{\mathbb{R}^{n}} G(z-y, t) d y=1, v_{0}(z) \in[0,1]$, and $|w(z, t)| \leq 1$ for all $(z, t) \in \mathbb{R}^{n} \times[0, \mathrm{~T}]$, it follows

$$
-1 \leq v(z, t) \leq \int_{\mathbb{R}^{n}} G(z-y, t)\left|v_{0}(y)\right| d y+|w(z, t)| \leq 2 .
$$

Thus, for any $(z, t) \in \mathbb{R}^{n} \times[0, T]$

$$
\begin{aligned}
|(\mathscr{F} w)(z, t)| & \leq \int_{0}^{t} \int_{\mathbb{R}^{n}} G(z-y, t-s)|f(v(y, s))| d y d s \\
& \leq \sup _{-1 \leq v \leq 2}|f(v)| T=1,
\end{aligned}
$$

which implies $\mathscr{F}$ maps $\mathscr{C}$ into itself. We now notice that for any $w_{1}, w_{2} \in \mathscr{C}$,

$$
\begin{aligned}
& \left|\left(\mathscr{F} w_{1}\right)(z, t)-\left(\mathscr{F} w_{2}\right)(z, t)\right| \\
& \quad \leq M \int_{0}^{t} \int_{\mathbb{R}^{n}}|G(z-y, t-s)|\left|w_{1}(y, s)-w_{2}(y, s)\right|^{\alpha} d y d s \\
& \quad \leq M T \sup _{z \in \mathbb{R}^{n}, t \in[0, T]}\left|w_{1}(x, t)-w_{2}(x, t)\right|^{\alpha},
\end{aligned}
$$

for some constant $M>0$ satisfying $\left|f(r)-\mathrm{f}\left(r^{\prime}\right)\right| \leq M$ $\left|r-r^{\prime}\right|^{\alpha}$ for any $r, r^{\prime} \in \mathbb{R}$, which means that the operator $\mathscr{F}$ is continuous from $\mathscr{C}$ into itself. Moreover, the image $\mathscr{F}(\mathscr{C})$ is relatively compact because $\mathscr{F}(\mathscr{C})$ is uniformly bounded for all $w \in \mathscr{C}$ and $\mathscr{F}(\mathscr{C})$ is equicontinuous by 
the estimates (30). Hence, by Tikhonov's fixed point theorem, there is $\widehat{w}(z, t) \in \mathscr{C}$ such that $\mathscr{F} \widehat{w}=\widehat{w}$. Recalling (39) and (40) gives that for all $(z, t) \in \mathbb{R}^{n} \times[0, T]$,

$$
\begin{aligned}
\widehat{v}(z, t):= & \int_{\mathbb{R}^{n}} G(z-y, t) v_{0}(y) d y+\widehat{w}(z, t) \\
= & \int_{\mathbb{R}^{n}} G(z-y, t) v_{0}(y) d y \\
& +\int_{0}^{t} \int_{\mathbb{R}^{\mathrm{n}}} G(z-y, t-s) f(\widehat{v}(y, s)) d y d s,
\end{aligned}
$$

which is a bounded mild solution to (21). Therefore, we obtain the existence of a bounded mild solution $v: \mathbb{R}^{n} \times$ $[0, \infty) \longrightarrow \mathbb{R}$ by replacing the initial time $t=0$ by $t=t_{0}$ for any $t_{0}>0$ and repeating the above procedure in each time interval $\left[t_{0}, t_{0}+T\right]$

\section{A Bounded Weak Solution and a Weak Comparison Principle}

In the previous section, we have proved that a mild solution to (21) enjoys regularity estimates for a classical solution. We now prove the uniqueness of the classical solution by showing that (21) possesses at most one weak solution. As a starting point, we define a weak $L^{\infty}$ sub- and supersolution of (21), and we then establish a comparison principle for them. The weak comparison principle concludes the uniqueness of a weak solution and plays an important role in the proof of stability in the next section.

Definition 5 (Weak $L^{\infty}$ sub- and supersolution). Let $v_{0}(z)$ $\in L^{\infty}\left(\mathbb{R}^{n}\right)$. By a weak $L^{\infty}$ supersolution of (21), we mean a function $\bar{v}(z, t) \in L^{\infty}\left(\mathbb{R}^{n} \times[0, \infty)\right)$ satisfying the following three conditions:

(i) For all nonnegative test function $\phi(z) \in W_{0}^{1,1}\left(\mathbb{R}^{n}\right)$,

$$
\underset{t \longrightarrow 0+}{\limsup } \int_{\mathbb{R}^{n}}\left[\bar{v}(z, t)-v_{0}(z)\right] \phi(z) d z \geq 0
$$

(ii) $\bar{v}$ is Lipschitz-continuous in every set $\mathbb{R}^{n} \times[\tau, T]$ whenever $0<\tau<T<\infty$, that is, $\partial \bar{v} / \partial z_{i}, \partial \bar{v} / \partial t \in L^{\infty}$ $\left(\mathbb{R}^{n} \times[\tau, T]\right)$ for all $i=1, \cdots, n$

(iii) For all nonnegative test function $\phi(z) \in W_{0}^{1,1}\left(\mathbb{R}^{n}\right)$,

$$
\begin{aligned}
& \int_{\mathbb{R}^{n}} \bar{v}_{t}(z, t) \phi(z) d z+\int_{\mathbb{R}^{n}} \nabla \bar{v}(z) \cdot \nabla \phi(z) d z \\
& -c \int_{\mathbb{R}^{n}} \bar{v}_{z_{1}}(z, t) \phi(z) d z \geq \int_{\mathbb{R}^{n}} f(\bar{v}(z, t)) \phi(z) d z
\end{aligned}
$$

By a weak $L^{\infty}$ subsolution of (21), we mean a function $\underline{v}(x, t) \in L^{\infty}\left(\mathbb{R}^{n} \times[0, \infty)\right)$ satisfying the above three conditions (i), (ii), and (iii) replaced the reverse inequality in (45) and (46). We say that a function $v \in L^{\infty}\left(\mathbb{R}^{n} \times[0, \infty)\right)$ is a weak $L^{\infty}$ solution of (21) if $v$ is a weak $L^{\infty}$ sub- and supersolution to (21).

We now establish a weak comparison principle for a weak $L^{\infty}$ sub- and supersolution under the crucial hypothesis (H3) which is one-sided Lipschitz continuity of $f$.

Theorem 6 (Weak comparison principle). Let $f: \mathbb{R} \longrightarrow \mathbb{R}$ be continuous with the one-sided Lipschitz condition (H3). Assume that $\underline{v}, \bar{v} \in L^{\infty}\left(\mathbb{R}^{n} \times[0, \infty)\right)$ are weak $L^{\infty}$ suband supersolutions to (21), respectively, such that $\underline{v}(z, 0)$ $\leq \bar{v}(z, 0)$ a.e. in $\mathbb{R}^{n}$. Then, we have $\underline{v}(z, t) \leq \bar{v}(z, t)$ a.e. in $\mathbb{R}^{n} \times(0, \infty)$.

Proof. Subtracting (46) from the reverse inequality of (46) for a subsolution yields

$$
\begin{aligned}
\int_{\mathbb{R}^{n}} & (\underline{v}-\bar{v})_{t} \phi d z+\int_{\mathbb{R}^{n}} \nabla(\underline{v}-\bar{v}) \cdot \nabla \phi d z-c \int_{\mathbb{R}^{n}}(\underline{v}-\bar{v})_{z_{1}} \phi d z \\
\leq & \int_{\mathbb{R}^{n}}[f(\underline{v})-f(\bar{v})] \phi d z,
\end{aligned}
$$

for any nonnegative function $\phi \in W_{0}^{1,1}\left(\mathbb{R}^{n}\right)$. Let $(\underline{v}-\bar{v})^{+}:=$ $\max \{(\underline{v}-\bar{v}), 0\}$, and we then prove that $(\underline{v}-\bar{v})^{+}=0$ a.e. in $\mathbb{R}^{n} \times(0, \infty)$. For any given nonnegative $\phi \in W_{0}^{1,1}\left(\mathbb{R}^{n}\right)$, since $(\underline{v}-\bar{v})^{+} \phi$ is also a nonnegative test function, replacing $\phi$ by $(\underline{v}-\bar{v})^{+} \phi$ in $(47)$ gives

$$
\begin{aligned}
& \int_{\mathbb{R}^{n}}(\underline{v}-\bar{v})_{t}(\underline{v}-\bar{v})^{+} \phi d z+\int_{\mathbb{R}^{n}} \nabla(\underline{v}-\bar{v}) \cdot \nabla\left[(\underline{v}-\bar{v})^{+} \phi\right] d z \\
& \quad-c \int_{\mathbb{R}^{n}}(\underline{v}-\bar{v})_{z_{1}}(\underline{v}-\bar{v})^{+} \phi \mathrm{d} z \leq \int_{\mathbb{R}^{n}}[f(\underline{v})-f(\bar{v})](\underline{v}-\bar{v})^{+} \phi d z .
\end{aligned}
$$

From a simple calculation, we evaluate that

$$
\int_{\mathbb{R}^{n}}(\underline{v}-\bar{v})_{t}(\underline{v}-\bar{v})^{+} \phi d z=\frac{1}{2} \frac{d}{d t} \int_{\mathbb{R}^{n}}\left[(\underline{v}-\bar{v})^{+}\right]^{2} \phi d z,
$$

$$
\begin{aligned}
\int_{\mathbb{R}^{n}} & \nabla(\underline{v}-\bar{v}) \cdot \nabla\left[(\underline{v}-\bar{v})^{+} \phi\right] d z \\
\quad= & \int_{\mathbb{R}^{n}}\left|\nabla(\underline{v}-\bar{v})^{+}\right|^{2} \phi d z+\frac{1}{2} \int_{\mathbb{R}^{n}} \nabla\left((\underline{v}-\bar{v})^{+}\right)^{2} \cdot \nabla \phi d z,
\end{aligned}
$$

$$
\int_{\mathbb{R}^{n}}(\underline{v}-\bar{v})_{z_{1}}(\underline{v}-\bar{v})^{+} \phi d z=\frac{1}{2} \frac{d}{d z_{1}} \int_{\mathbb{R}^{n}}\left[(\underline{v}-\bar{v})^{+}\right]^{2} \phi d z,
$$

and

$$
\int_{\mathbb{R}^{n}}[f(\underline{v})-f(\bar{v})](\underline{v}-\bar{v})^{+} \phi d z \leq L \int_{\mathbb{R}^{n}}\left[(\underline{v}-\bar{v})^{+}\right]^{2} \phi d z,
$$


where the last inequality is from the one-sided Lipschitz condition (H3). Hence, we find

$$
\begin{aligned}
& \frac{1}{2} \frac{d}{d t} \int_{\mathbb{R}^{n}}\left[(\underline{v}-\bar{v})^{+}\right]^{2} \phi d z+\frac{1}{2} \int_{\mathbb{R}^{n}} \nabla\left(\left[(\underline{v}-\bar{v})^{+}\right]^{2}\right) \\
& \quad \cdot \nabla \phi d z+\int_{\mathbb{R}^{n}}\left|\nabla(\underline{v}-\bar{v})^{+}\right|^{2} \phi d z-\frac{c}{2} \frac{d}{d z_{1}} \int_{\mathbb{R}^{n}}\left[(\underline{v}-\bar{v})^{+}\right]^{2} \phi d z \\
& \leq L \int_{\mathbb{R}^{n}}\left[(\underline{v}-\bar{v})^{+}\right]^{2} \phi d z .
\end{aligned}
$$

By multiplying each term of (50) by $2 e^{-2 L t}$ and rearranging, we obtain

$$
\begin{aligned}
& e^{-2 L t} \frac{d}{d t} \int_{\mathbb{R}^{n}}\left[(\underline{v}-\bar{v})^{+}\right]^{2} \phi d z-2 L e^{-2 L t} \int_{\mathbb{R}^{n}}\left[(\underline{v}-\bar{v})^{+}\right]^{2} \phi d z \\
& +e^{-2 L t} \int_{\mathbb{R}^{n}} \nabla\left(\left[(\underline{v}-\bar{v})^{+}\right]^{2}\right) \cdot \nabla \phi d z \\
& +c e^{-2 L t} \frac{d}{d z_{1}} \int_{\mathbb{R}^{n}}\left[(\underline{v}-\bar{v})^{+}\right]^{2} \phi d z \\
& +2 e^{-2 L t} \int_{\mathbb{R}^{n}}\left|\nabla(\underline{v}-\bar{v})^{+}\right|^{2} \phi d z \leq 0 .
\end{aligned}
$$

By setting $W(z, t)=e^{-2 L t}\left[(\underline{v}-\bar{v})^{+}\right]^{2}$, it follows that

$$
\begin{aligned}
& \int_{\mathbb{R}^{n}} W_{t} \phi d z+\int_{\mathbb{R}^{n}} \nabla W \cdot \nabla \phi d z-c \int_{\mathbb{R}^{n}} W_{z_{1}} \phi d z \\
& \quad+2 \int_{\mathbb{R}^{n}}|\nabla W|^{2} \phi d z \leq 0 .
\end{aligned}
$$

Since $\int_{\mathbb{R}^{n}} \nabla W \cdot \nabla \phi d z=-\int_{\mathbb{R}^{n}} \Delta W \phi d z$ and $\int_{\mathbb{R}^{n}}|\nabla W|^{2} \phi d z$ $\geq 0$, we find

$$
\int_{\mathbb{R}^{n}} W_{t} \phi d z-\int_{\mathbb{R}^{n}} \Delta W \phi d z-c \int_{\mathbb{R}^{n}} W_{z_{1}} \phi d z \leq 0,
$$

for all nonnegative $\phi \in W_{0}^{1,1}\left(\mathbb{R}^{n}\right)$. As $\underline{v}(z, 0) \leq \bar{v}(z, 0)$ a.e. in $\mathbb{R}^{n}, W(z, 0)=0$ a.e. in $\mathbb{R}^{n}$ and thus $W(z, t)$ satisfies the initial value problem

$$
\left\{\begin{array}{l}
W_{t}-\Delta_{z} W-c W_{z_{1}} \leq 0 \\
W(z, 0)=0
\end{array}\right.
$$

By setting $V\left(z_{1}, z_{2}, \cdots, z_{n}, t\right)=W\left(z_{1}-c t, z_{2}, \cdots, z_{n}, t\right)$,

$$
\left\{\begin{array}{l}
V_{t}-\Delta V \leq 0 \\
V(z, 0)=0
\end{array}\right.
$$

The weak maximum principle for the heat equation concludes that $W(z, t) \leq 0$ a.e. in $\mathbb{R}^{n} \times(0, \infty)$, which implies $(\underline{v}-\bar{v})^{+} \equiv 0$ a.e. in $\mathbb{R}^{n} \times(0, \infty)$. Therefore,

$$
\underline{v}(z, t) \leq \bar{v}(z, t) \text { a.e.in } \mathbb{R}^{n} \times(0, \infty) .
$$

Since any bounded weak solution is a bounded sub- and supersolution simultaneously, as a consequence of the weak comparison principle together with Lemmas 3 and 4, we obtain the following corollary.

Corollary 7. Let $f: \mathbb{R} \longrightarrow \mathbb{R}$ be continuous satisfying (H2) and (H3). Then the initial value problem (21) has a unique bounded classical solution.

\section{Lyapunov Stability of the Planar Wave $U$}

In this section, we give a proof of Theorem 1 . We prove the planar wave $U$ is stable in the sense that the unique solution $v(z, t)$ to the initial value problem (21) stays near $U$ for all time $t>0$ when the initial data $v_{0}(z)$ at $t=0$ is close enough to $U$.

We first construct, by an appropriate modification of the planer wave $U\left(z_{1}\right)$, weak sub- and supersolutions $\underline{v}(z, t)$ and $\bar{v}(z, \mathrm{t})$, respectively, to $(21)$ satisfying

$$
\underline{v}(z, 0) \leq v_{0}(z) \leq \bar{v}(z, 0) \text { and } 0 \leq \underline{v}(z, t) \leq \bar{v}(z, t) \leq 1,
$$

for all $(z, t) \in \mathbb{R}^{n} \times(0, \infty)$. The assumption (H4) plays a significant role in the construction of the sub- and supersolution. We notice that the constant functions $v(z, t)=0$ and $v(z, t)=1$ are sub- and supersolutions, respectively, to (21) because the initial data $v_{0}(z)$ satisfies $0 \leq v_{0}(z) \leq 1$ for all $z$ $\in \mathbb{R}^{n}$.

Lemma 8. Suppose that $f$ satisfies the assumptions (H1)-(H4) and the initial data $v_{0}(z)$ of (21) satisfies for all $z \in \mathbb{R}^{n}$

$$
0 \leq v_{0}(z) \leq 1 \text { and }\left|v_{0}(z)-U\left(z_{1}\right)\right|<E_{0} \text {, }
$$

where $E_{0}>0$ is sufficiently small. Then, there exists a constant $v>0$ such that the functions defined by

$$
\begin{aligned}
& \underline{v}(z, t)=\max \left\{U\left(z_{1}-\xi(t)\right)-q(t), 0\right\} \text { and } \\
& \bar{v}(z, t)=\min \left\{U\left(z_{1}+\xi(t)\right)+q(t), 1\right\},
\end{aligned}
$$

where

$q(t)=2 E_{0} e^{-\mu t}, \mu=\min \{\underline{\mu}, \bar{\mu}\} \quad$ and $\quad \xi(t)=2 \nu E_{0}\left(1-e^{-\mu t}\right)$

are sub- and supersolutions, respectively, to (21) satisfying (60) (see Figure 2). 


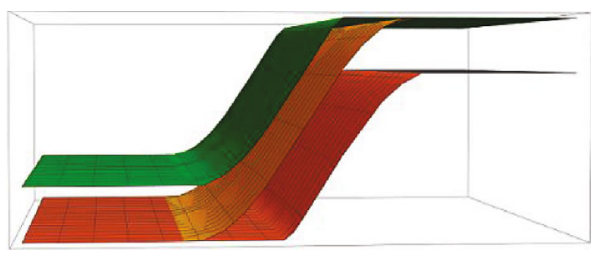

(a) $t=0$

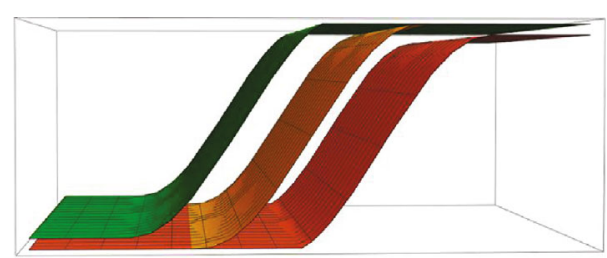

(b) $t>0$

Figure 2: The subsolutions (red) and supersolutions (green) given in (62)-(63) by a modification of the planar wave $U$ (yellow) in $\mathbb{R}^{2}$. If the initial data $v_{0}(z)$ is close enough to $U$ so that $v_{0}(z)$ is between sub- and supersolutions at $t=0$, the solution $v(z, t)$ stays between them for all $t>0$. The fact that both $v$ and $U$ are trapped by the sub- and supersolutions whose difference is sufficiently small for all time $t>0$ guarantees the stability of $U$.

Proof. To begin, we recall the profile $U\left(z_{1}\right)$ in (12) and let $E_{0}$ be sufficiently small that

$$
0<E_{0}<\eta_{0}<\frac{1}{4} \min \left\{s_{0}, 1-s_{0}\right\}
$$

where $s_{0}$ is a constant such that $U(0)=s_{0}$. Then, by the assumption (61) for an initial data $v_{0}$, one can say that for all $z \in \mathbb{R}^{n}$,

$0 \leq \max \left\{U\left(z_{1}\right)-2 E_{0}, 0\right\} \leq v_{0}(z) \leq \min \left\{U\left(z_{1}\right)+2 E_{0}, 1\right\} \leq 1$,

which implies that

$$
\underline{v}(z, 0) \leq v_{0}(z) \leq \bar{v}(z, 0) .
$$

We notice that $q(t)$ is decreasing to 0 and $\xi(t)$ is increasing to $2 v E_{0}$ as $t \longrightarrow \infty$, where the positive constant $v$ will be determined later. Moreover, by the monotonicity of the profile $U\left(z_{1}\right)$ and the inequality (66), the functions $\underline{v}$ and $\bar{v}$ satisfy (60).

By setting $\eta:=E_{0}<\eta_{0}$ in the assumption (H4), we prove $\bar{v}(z, t)$ is a supersolution to (21). In order to prove $\bar{v}(z, t)$ : $\mathbb{R}^{n} \times[0, \infty) \longrightarrow \mathbb{R}$ is a supersolution to (21), we decompose its domain as

$$
\mathbb{R}^{n} \times[0, \infty)=\bar{\Omega}_{1} \cup \bar{\Omega}_{2} \cup \bar{\Omega}_{3},
$$

where

$$
\begin{aligned}
& \bar{\Omega}_{1}=\left\{(z, t) \in \mathbb{R}^{n} \times[0, \infty) \mid 0 \leq U\left(z_{1}+\xi(t)\right) \leq \delta\right\}, \\
& \bar{\Omega}_{2}=\left\{(z, t) \in \mathbb{R}^{n} \times[0, \infty) \mid 1-\delta \leq U\left(z_{1}+\xi(t)\right) \leq 1\right\}, \\
& \bar{\Omega}_{3}=\left\{(z, t) \in \mathbb{R}^{n} \times[0, \infty) \mid \delta \leq U\left(z_{1}+\xi(t)\right) \leq 1-\delta\right\},
\end{aligned}
$$

with the positive constant $\delta \leq E_{0}$ in the assumption (H4). That is, the decomposition is depending only on the $z_{1}$-coordinate and $t$.

For the region $\bar{\Omega}_{1}$, since $q(t)$ is decreasing and by (64),

$U\left(z_{1}+\xi(t)\right)+q(t) \leq U\left(z_{1}+\xi(t)\right)+q(0) \leq \delta+2 E_{0} \leq 3 E_{0}<1$, and the profile equation $U^{\prime \prime}+c U^{\prime}+f(U)=0$ gives

$$
\begin{aligned}
\bar{v}_{t}-\Delta \bar{v}-c \bar{v}_{z_{1}}-f(\bar{v})= & U^{\prime}\left(z_{1}+\xi(t)\right) v q^{\prime}(t)+q^{\prime}(t) \\
& -U^{\prime \prime}\left(z_{1}+\xi(t)\right)-c U^{\prime}\left(z_{1}+\xi(t)\right) \\
& -f\left(U\left(z_{1}+\xi(t)\right)+q(t)\right) \\
= & U^{\prime}\left(z_{1}+\xi(t)\right) v q^{\prime}(t)+q^{\prime}(t) \\
& +f\left(U\left(z_{1}+\xi(t)\right)\right) \\
& -f\left(U\left(z_{1}+\xi(t)\right)+q(t)\right) .
\end{aligned}
$$

Since $0 \leq U \leq \delta$ and $0<q(t) \leq q(0)=2 E_{0}<s_{0}-2 E_{0}=s_{0}$ $-2 \eta$ for all $(z, t) \in \bar{\Omega}_{1}$, applying the inequality (16) to the last two terms on the right-hand side of (73) and recalling $U^{\prime} \geq 0$ and $\mu=\min \{\mu, \bar{\mu}\}$ yield

$$
\begin{aligned}
\bar{v}_{t}-\Delta \bar{v}-c \bar{v}_{z_{1}}-f(\bar{v}) & \geq U^{\prime}\left(z_{1}+\xi(t)\right) v q^{\prime}(t)+q^{\prime}(t)+\underline{\mu q}(t) \\
& =U^{\prime}\left(z_{1}+\xi(t)\right) v \mu q(t)-\mu q(t)+\underline{\mu q}(t) \\
& =q(t)\left[v \mu U^{\prime}-\mu+\bar{\mu}\right] \geq 0,
\end{aligned}
$$

which implies $\bar{v}(z, t)$ is a supersolution to (21) on $\bar{\Omega}_{1}$.

For the region $\bar{\Omega}_{2} \cup \bar{\Omega}_{3}$, by the definition of $\bar{v}$ and the fact that $v \equiv 1$ is a supersolution to (21), it is enough to prove $\bar{v}$ is a supersolution only when $U\left(z_{1}+\xi(t)\right)+q(t)<1$. Since $0<q(t) \leq q(0)=2 E_{0}<1-s_{0}-2 E_{0}=1-s_{0}-2 \eta$ for all $(z, t)$ $\in \bar{\Omega}_{2}$, applying (17) to the last two terms on the righthand side of (73) gives

$$
\begin{aligned}
\bar{v}_{t}-\Delta \bar{v}-c \bar{v}_{z_{1}}-f(\bar{v}) & \geq U^{\prime}\left(z_{1}+\xi(t)\right) v q^{\prime}(t)+q^{\prime}(t)+\bar{\mu} q(t) \\
& =q(t)\left[v \mu U^{\prime}-\mu+\bar{\mu}\right] \geq 0 .
\end{aligned}
$$

For all $(z, t) \in \bar{\Omega}_{3}$, applying the assumption (H3) to the last two terms on the right-hand side of (73) yields 


$$
\begin{aligned}
\bar{v}_{t}-\Delta \bar{v}-c \bar{v}_{z_{1}}-f(\bar{v}) & \geq U^{\prime}\left(z_{1}+\xi(t)\right) v q^{\prime}(t)+q^{\prime}(t)-L q(t) \\
& =q(t)\left[v \mu U^{\prime}-\mu-L\right] .
\end{aligned}
$$

Since the profile $U$ is a strictly increasing $C^{2}$-function for $\delta \leq U \leq 1-\delta$, there exists some $\omega>0$ such that $U^{\prime} \geq \omega$ for all $(z, t) \in \bar{\Omega}_{3}$. If we choose $v$ satisfying $v \geq \mu+L / \mu \omega$, we obtain

$$
\bar{v}_{t}-\Delta \bar{v}-c \bar{v}_{z_{1}}-f(\bar{v}) \geq q(t)[\nu \mu \omega-\mu-L] \geq 0,
$$

which implies $\bar{v}$ is a supersolution to (21). The proof of the subsolution $\underline{v}(z, t)$ follows similarly by decomposing $\mathbb{R}^{n} \times[$ $0, \infty)$ as

$$
\mathbb{R}^{n} \times[0, \infty)=\underline{\Omega}_{1} \cup \underline{\Omega}_{2} \cup \underline{\Omega}_{3},
$$

where

$$
\begin{aligned}
& \underline{\Omega}_{1}=\left\{(z, t) \in \mathbb{R}^{n} \times[0, \infty) \mid 1-\delta \leq U\left(z_{1}-\xi(t)\right) \leq 1\right\}, \\
& \underline{\Omega}_{2}=\left\{(z, t) \in \mathbb{R}^{n} \times[0, \infty) \mid 0 \leq U\left(z_{1}-\xi(t)\right) \leq \delta\right\}, \\
& \underline{\Omega}_{3}=\left\{(z, t) \in \mathbb{R}^{n} \times[0, \infty) \mid \delta \leq U\left(z_{1}-\xi(t)\right) \leq 1-\delta\right\},
\end{aligned}
$$

and using (17), (16), and (H3) for $\underline{\Omega}_{1}, \underline{\Omega}_{2}$, and $\underline{\Omega}_{3}$, respectively. $\square$

Remark 9. Similarly as in [6], one can also construct suband supersolutions satisfying (60) even if the initial data is close enough to $U$ only at space infinity in $\mathbb{R}^{n}$ (possibly large initial perturbations). In that case, $\xi(0)$ might be strictly positive, while $\xi(0)=0$ in our case. However, the initial perturbation in [6] is not enough to obtain stability results of the planar wave in $\mathbb{R}^{n}$. Thus, we prove the stability under small initial perturbations (61) and so simply set $\xi(0)=0$.

We now prove the main theorem.

Proof of Theorem 1 . Let $E_{0}$ be sufficiently small that

$$
0<E_{0}<\eta_{0}<\frac{1}{4} \min \left\{s_{0}, 1-s_{0}\right\}
$$

By Lemma 8, the sub- and supersolutions $\underline{v}(z, t)$ and $\bar{v}(z, t)$ to (21) defined by (62)-(63) satisfy

$$
\underline{v}(z, t) \leq U\left(z_{1}\right) \leq \bar{v}(z, t) .
$$

Moreover, since the initial data $v_{0}$ satisfies $\underline{v}(z, 0) \leq v_{0}$ $(z) \leq \bar{v}(z, 0)$, by the weak comparison principle in the previous section, the unique bounded classical solution $v(z, t)$ to (21) also satisfies

$$
\underline{v}(z, t) \leq v(z, t) \leq \bar{v}(z, t) .
$$

Therefore, recalling (63) and $U$ is a $C^{2}$-profile satisfying (12), the inequalities (78) and (79) yield that for all $(z, t) \in \mathbb{R}^{n} \times(0, \infty)$,

$$
\begin{aligned}
\left|v(z, t)-U\left(z_{1}\right)\right| & \leq|\bar{v}(z, t)-\underline{v}(z, t)| \\
& =\left|U\left(z_{1}+\xi(t)\right)+q(t)-U\left(z_{1}-\xi(t)\right)+q(t)\right| \\
& \leq 2 \xi(t) \sup _{z_{1} \in \mathbb{R}}\left|U^{\prime}\left(z_{1}\right)\right|+2 q(t) \\
& \leq 4 v E_{0}\left(1-e^{-\mu t}\right) \sup _{z_{1} \in \mathbb{R}}\left|U^{\prime}\left(z_{1}\right)\right|+4 E_{0} e^{-\mu t} \\
& \leq C E_{0},
\end{aligned}
$$

for some constant $C>0$.

\section{Data Availability}

The results of our manuscript are not based on data but only mathematical analysis.

\section{Conflicts of Interest}

The authors declare that they have no conflicts of interest.

\section{Acknowledgments}

This work was supported by the research grant of the Kongju National University in 2019. The research of the first author was supported by the National Research Foundation of Korea (NRF) grant funded by the Korea government (MSIP) (no. 2019R1F1A1063018).

\section{References}

[1] P. C. Fife and J. B. McLeod, "The approach of solutions of nonlinear diffusion equations to travelling front solutions," Archive for Rational Mechanics and Analysis, vol. 65, no. 4, pp. 335-361, 1977.

[2] J. D. Murray, Mathematical Biology I: An Introduction, in Interdisciplinary Applied Mathematics, vol. 17, Springer-Verlag, Berlin-Heidelberg-New York, 2002.

[3] H. Matano, M. Nara, and M. Taniguchi, "Stability of planar waves in the Allen-Cahn equation," Communications in Partial Differential Equations, vol. 34, no. 9, pp. 976-1002, 2009.

[4] R. A. Fisher, "The wave of advance of advantageous genes," Annals of eugenics, vol. 7, no. 4, pp. 355-369, 1937.

[5] W. H. Fleming, "A selection-migration model in population genetics," Journal of Mathematical Biology, vol. 2, no. 3, pp. 219-233, 1975.

[6] P. Drábek and P. Takáč, "Convergence to travelling waves in Fisher's population genetics model with a non-Lipschitzian reaction term," Journal of mathematical biology, vol. 75, no. 4, pp. 929-972, 2017.

[7] A. Tsoularis and J. Wallace, "Analysis of logistic growth models," Mathematical Biosciences, vol. 179, no. 1, pp. 21-55, 2002.

[8] M. Taniguchi and M. Nara, "Stability of a traveling wave in curvature flows for spatially non-decaying initial perturbations," 
Discrete \& Continuous Dynamical Systems, vol. 14, no. 1, pp. 203-220, 2006.

[9] P. Drábek and P. Takáč, "New patterns of travelling waves in the generalized Fisher-Kolmogorov equation," Nonlinear Differential Equations and Applications NoDEA, vol. 23, no. 2, 2016.

[10] O. A. Ladyzhenskaya, N. N. Ural'tseva, and V. A. Solonnikov, Linear and quasilinear equations of parabolic type, vol. 23, American Mathematical Soc, 1988. 